\title{
Aerodynamic Free-Flight Conditions in Wind Tunnel Modelling through Reduced-Order Wall Inserts
}

\author{
Narges Tabatabaei *(D), Ramis Örlü (D), Ricardo Vinuesa (D) and Philipp Schlatter (D) \\ SimEx/FLOW, Engineering Mechanics, KTH Royal Institute of Technology, 10044 Stockholm, Sweden; \\ ramis@mech.kth.se (R.Ö.); rvinuesa@mech.kth.se (R.V.); pschlatt@mech.kth.se (P.S.) \\ * Correspondence: nargest@kth.se
}

Citation: Tabatabaei, N.; Örlü, R.;

Vinuesa, R.; Schlatter, P. Aerodynamic Free-Flight Conditions in Wind Tunnel Modelling through Reduced-Order Wall Inserts. Fluids 2021, 6, 265. https://doi.org/ $10.3390 /$ fluids 6080265

Academic Editor: Markus Klein

Received: 28 May 2021

Accepted: 21 July 2021

Published: 27 July 2021

Publisher's Note: MDPI stays neutral with regard to jurisdictional claims in published maps and institutional affiliations.

Copyright: (c) 2021 by the authors. Licensee MDPI, Basel, Switzerland. This article is an open access article distributed under the terms and conditions of the Creative Commons Attribution (CC BY) license (https:// creativecommons.org/licenses/by/ $4.0 /)$.

\begin{abstract}
Parallel sidewalls are the standard bounding walls in wind tunnels when making a wind tunnel model for free-flight condition. The consequence of confinement in wind tunnel tests, known as wall-interference, is one of the main sources of uncertainty in experimental aerodynamics, limiting the realizability of free-flight conditions. Although this has been an issue when designing transonic wind tunnels and/or in cases with large blockage ratios, even subsonic wind tunnels at low-blockage-ratios might require wall corrections if a good representation of free-flight conditions is intended. In order to avoid the cumbersome streamlining methods especially for subsonic wind tunnels, a sensitivity analysis is conducted in order to investigate the effect of inclined sidewalls as a reduced-order wall insert in the airfoil plane. This problem is investigated via Reynolds-averaged Navier-Stokes (RANS) simulations, and a NACA4412 wing at the angles of attack between 0 and 11 degrees at a moderate Reynolds number $(400 \mathrm{k})$ is considered. The simulations are validated with well-resolved large-eddy simulation (LES) results and experimental wind tunnel data. Firstly, the wallinterference contribution in aerodynamic forces, as well as the local pressure coefficients, are assessed. Furthermore, the isolated effect of confinement is analyzed independent of the boundary-layer growth. Secondly, wall-alignment is modified as a calibration parameter in order to reduce wallinterference based on the aforementioned assessment. In the outlined method, we propose the use of linear inserts to account for the effect of wind tunnel walls, which are experimentally simple to realize. The use of these inserts in subsonic wind tunnels with moderate blockage ratio leads to very good agreement between free-flight and wind tunnel data, while this approach benefits from simple manufacturing and experimental realization.
\end{abstract}

Keywords: CFD; RANS; wind tunnel; wall interference; free-flight; confinement; insert wall

\section{Introduction}

The configuration of wind-tunnel sidewalls affects the pressure-gradient distributions over wind-tunnel models [1-6]. An early example of wind tunnel testing limitation is documented in 1938 for experiments in conditions close to the speed of sound, where the results of the wind tunnel test did not correspond to the practical case of a body moving through still air [7]. The occurrence of the compression shock in the wind tunnel, which was later denoted as 'transonic blockage', was resolved based on the principle of wall streamlining [8]. In this method, a streamline is selected to separate the flow around the wing and the theoretical infinite region encountered in free-flight conditions, thereby the real infinite flow field is split into two parts with a streamtube (ignoring the boundary-layer (BL) growth on the test-section walls) [9].

The wind tunnel walls are modified (typically through inserts) such that they follow a particular streamline out of the BL region. The idea of streamlining has also been used to address large blockage ratio issues [10]. A nonflexible streamlined wind tunnel, where inserts are utilized within the test section, was used rather than a parallel-walled tunnel. Stafford [10] stated that mathematical techniques could be used to determine 
the optimum wall geometry for any specific test body, but that the tunnel should have been equipped with movable walls to do so. The adaptive-wall method was introduced as a technique to implement the concept of streamlining in transonic testing. In 1975, the reduction of wall-interference was assessed in a low speed self-streamlining wind tunnel [11] with the purpose of designing a transonic test section. Although the experiment used a large number of jacks for three-dimensional (3D) testing, the correction method was not successful near the stall region. For steady subsonic flows, theories of lift and blockage interference have been developed from 1966 for two-dimensional (2D) and 3D cases [12]. Similar to the streamlining method, the formulations resulted in adequate corrections for flows without separation. In 1984, a contoured wind tunnel liner was used to alleviate tunnel interference in transonic-flow conditions based on the concept of 'streamlining' wind tunnel walls [13]. The designed liner included a contraction cone and a diffuser (chock) section upstream and downstream of the test section, respectively.

Considering the limitations of adaptive walls and other correction methods, an engineering solution is required to bridge the gap between academic and industrial interests, while it is also efficient for separated flows. The alignment of ceiling-walls have been used as a calibration factor, e.g., in tuning a required boundary layer condition via a flexible ceiling $[14,15]$. The idea of this paper is to apply a similar idea in the airfoil plane and reduce the wall-interference with inclined side-walls. In subsonic wind tunnels with low blockage ratio, the wall interface is limited and the wall insert can be designed in a more practical way. The feasibility of such a method is investigated in this work via a sensitivity analysis. Despite all the advantages of adaptive walls in both small-scale and large-scale facilities, they were recognized to be too complex from an implementation point of view, in addition to the observed insufficiency for near-stall conditions [16]. In recent years, wall-interference effects are mainly treated with blockage-correction methods for either full-scale models or the typical wind-tunnel wing models [17,18].

This article characterizes the aerodynamic properties of a NACA4412 wing section, comparing the results of wind tunnel tests with those that correspond to free-flight conditions, obtained through numerical simulation. The NACA4412 is selected since it is a common test case for numerical and experimental studies [19-21]. The numerical work includes Reynolds-Averaged Navier-Stokes (RANS) simulations, validated with wellresolved large-eddy simulations (LES). Here, we also introduce and assess a new procedure to design wall inserts that aims at compensating the confinement in the airfoil plane, where we consider a linear geometry adjusted in order to improve the agreement between the experimental data and the free-flight conditions. The advantages of the outlined method are its simplicity of the insert-manufacturing process, as well as their installation in the test section. This also avoids manufacturing complex insert geometries or employing experimental facilities with adaptive walls.

The article is structured as follows: The setup is described in Section 2, which includes the details of the simulation setup and the mesh analysis. The validity of the numerical simulation is analyzed in Section 3, including both the confined and unconfined flow fields. The different categories of wall effects is described in Section 4.1, along with the specific approach of this paper in Section 4.2. Then, the method implementation is outlined in Section 5 comprising the results of the proposed approach in terms of the different parameters.

\section{Simulation Setup}

This study investigates a method to improve the agreement between free-flight conditions and a wind tunnel model of a wing with a constant cross section at low-speed. We focus on the aerodynamics of a NACA4412 wing section. The facility under consideration is the Minimum-Turbulence-Level (MTL) wind tunnel, which is a closed-loop circuit tunnel housed at KTH. Additional information about the MTL wind tunnel can be found in Ref. [22], while the main characteristics are summarized as follows. The test section is $7 \mathrm{~m}$ long and has a cross-sectional area of $1.2 \times 0.8 \mathrm{~m}^{2}$. The closed-loop configuration of 
the MTL provides stable velocity and temperature conditions for the BL measurements. The maximum Mach number for the implemented experiments is $\approx 0.2$ in the test section. With a vertical wing located at the middle of the test section, the blockage ratio is approximately $5 \%$ at zero degree angle of attack (AOA). An illustration of the wind tunnel test section is shown in Figure 1.

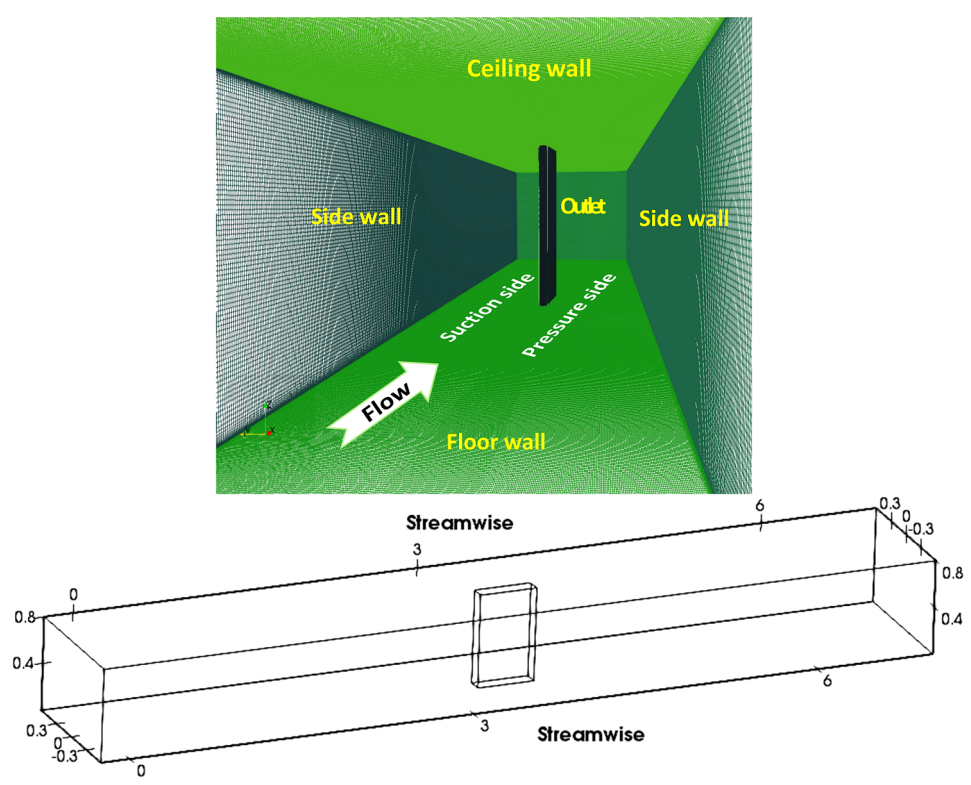

Figure 1. Illustration of the wind tunnel test section, where the flow is in positive $x$ direction.

Computational fluid dynamics CFD is our tool to model both free-flight conditions and wind tunnel models. The majority of the investigations for the different AOAs are based on 2D simulations at the mid-height plane in the wind tunnel; however, we will also perform 3D simulations of the complete wind tunnel for selected cases.

The RANS simulations are carried out with finite volume discretization via the opensource OpenFOAM code with the SIMPLE (semi-implicit method for pressure linked equations) and PIMPLE (merged PISO-SIMPLE) algorithms, where PISO stands for pressureimplicit with splitting of operators [23]. Second order Gaussian integration was selected for the gradient terms. Turbulence and wall-treatment are modeled based on the $k-\omega$ shear-stress transport (SST) model [24] and the first grid point in the wall-normal direction is always located at a distance from the wall below one viscous unit (i.e., in terms of the viscous length scale $\ell_{*}=v / u_{\tau}$, where $v$ and $u_{\tau}$ are the fluid kinematic viscosity and friction velocity, respectively). Appropriate wall functions are set over the walls for different properties. Zero-gradient boundary conditions are applied for the outflow, while the inflow conditions are defined with the fixed values according to the realistic turbulence intensity (Tu) for a high-quality wind tunnel $(\mathrm{Tu}=0.1 \%)$.

In order to make a numerical model, the wind tunnel test section was meshed using a multi-block structured hexahedral mesh and the geometry was created using ANSYS ICEM CFD [25]. The 2D domain with one-layer of cells was built to represent the middle cross section of the wind tunnel, i.e., the section at the wing mid-height, where the full-scale 3D computational domain was generated according to the wind-tunnel dimensions. Ceiling and floor are assumed to be unchanged, hence the method does not aim at achieving infinite span conditions. Here, instead, the investigations comprise the modifications on the two sidewalls, see Figure 1, thereby aiming at reducing the wall interference in the airfoil plane. In the 3D simulations, the modified sidewalls are extruded along the span.

\subsection{Mesh Validation}

In this section, we describe the mesh-sensitivity analysis. With the purpose of avoiding wall-functions near the wall, the first grid point is fixed in the mesh analysis process. 
The arrangement of the mesh blocks, and the relative bunching of the cells was implemented considering the quality parameters (e.g., volume change), as well as the required refinement at the critical parts (e.g., over the suction side of the airfoil). In order to investigate the adequacy of the grid resolution, we assessed the mesh at the near-stall angle to ensure the mesh-independence, as the worst-case scenario, and thus ensure a sufficient mesh resolution also at all lower angles of attack.

Figure 2-left, shows the overall resolution of six analyzed grids, with a local refinement on the airfoil from D3 to D1, and a global refinement from D1 to A. Table 1 summarizes some features of every grid, including the maximum aspect ratio among the cells over the suction side of the airfoil. The plots in Figure 2-mid and right, show the sensitivity of the solution to these meshes, according to relevant integral and local quantities, respectively. The errors are calculated with respect to the results of the most refined mesh, A. The reported Quality in Table 1 is a weighted diagnostic between Determinant (between -1 and 1), Max Orthogls (normalized between -1 and 1) and Max Warpgls (normalized between 0 and 1) [26]. More information about these mesh diagnostics is available in Ref. [26]. In the coarsest mesh, D3, $99.7 \%$ of the cells have a high quality $(>0.9)$, that indicates appropriate base blocking and bunching. Although the resolution of grid B already provides a reliable result, we decided to use the more refined grid $\mathrm{A}$ in this paper, considering a safety margin to study the various of simulation cases in the later part of the paper.
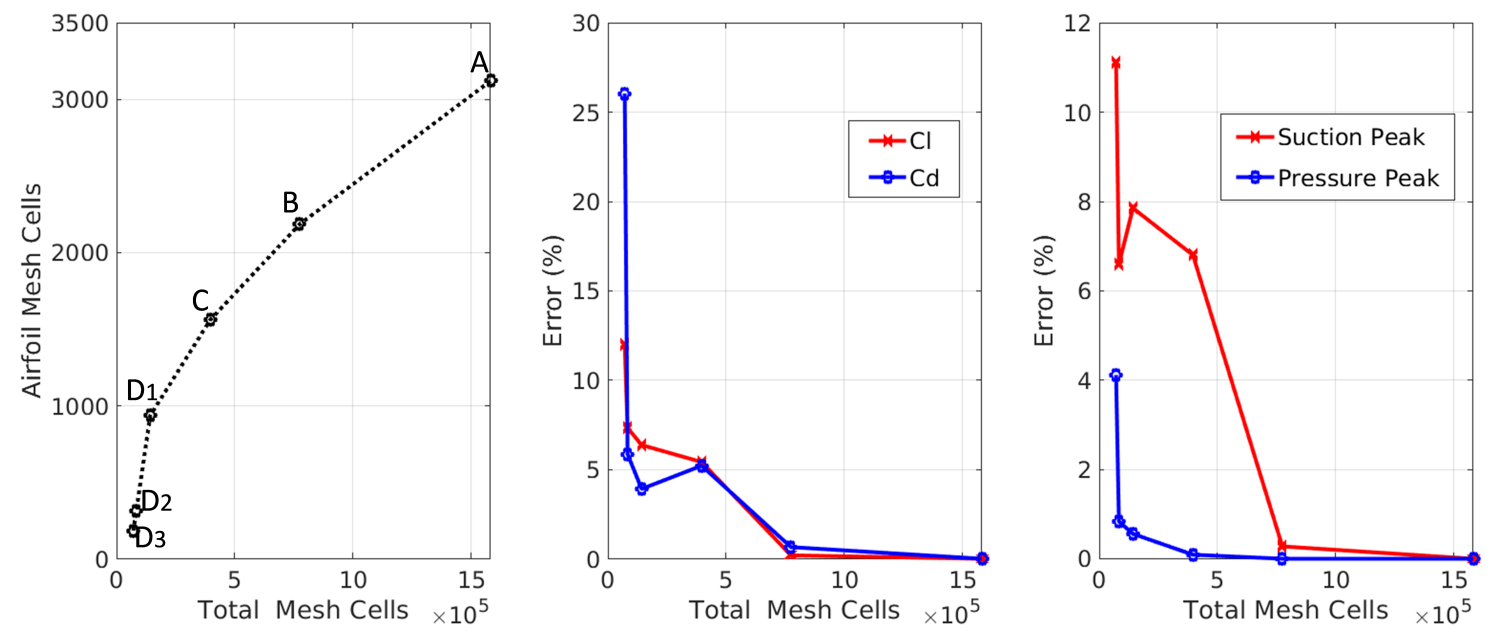

Figure 2. Mesh resolution analysis; A, B, C, D1, D2 and D3 are the tested grids with more detailed information given in Table 1. Note that for all panels, the convergence is monotonic in terms of total mesh cells.

Table 1. Mesh quality analysis; Quality ranges from 0 to 1 for the worst and the best quality, respectively.

\begin{tabular}{ccccccc}
\hline METRIC & A & B & C & D1 & D2 & D3 \\
\hline Minimum Quality in the whole domain & 0.93 & 0.91 & 0.83 & 0.60 & 0.59 & 0.59 \\
\hline Number of elements with Quality $>0.9$ in [\%] & 100 & 100 & 99.98 & 99.7 & 99.7 & 99.7 \\
\hline Maximum aspect ratio over the airfoil suction side & 8 & 12 & 17 & 29 & 96 & 192 \\
\hline
\end{tabular}

In addition to the mesh sensitivity analysis, our data on the final mesh will be validated against the experimental results obtained in the wind tunnel. These data will be presented later in the paper (Section 3).

In order to represent the unconfined flow in free-flight conditions, an airfoil is modeled in a large computational domain. A circular domain with a diameter of $50 \mathrm{C}$ is considered, where $C$ is the chord length. For the free-flight conditions, a structured hexahedral mesh is generated with the open-source meshing software Gmsh [27]. The Reynolds number based on freestream velocity $U_{\infty}$ and $C$ is 400,000 for all the cases, which corresponds to that of 
our previous direct-numerical-simulation (DNS) work [28]. The wing is tripped on the suction and pressure sides at $x / C=0.1$, where $x$ is the axial location from the leading edge (LE), as is the case for the corresponding model experiment as well as the LES reference data. The tripping methods are based on Refs. [19,21] for LES and Ref. [29] for the RANS. The mesh resolution for the free-flight cases on the wing surface is chosen to reflect a mesh between $A$ and $B$ as in the previous analysis. In addition to the mesh sensitivity as given in the current section, we will also present a validation against LES data later in Section 3.

\section{Simulation Validation}

The pressure distribution is considered as the main parameter for the validation because it is the most important factor to determine the aerodynamics. Static pressure, $p$, is non-dimensionalized with the dynamic pressure $P_{d}$ and the resultant coefficient, $C_{p}$, is commonly defined such that it equals unity at the stagnation point $\left(C_{p_{s t a g}}=1\right)$ : $C_{p}=\left(p-p_{\infty}\right) / P_{d}$.

\subsection{Free-Flight Condition (Unconfined Flow)}

With the setup mentioned in Section 2.1, the unconfined flow is simulated. The qualitative velocity contour is shown in Figure 3, further discussed in Section 4.1.1. Pressure distribution, via the pressure coefficient $C_{p}$, is plotted over the suction and the pressure sides from leading edge $(\mathrm{LE}, x / C=0)$ to the TE $(x / C=1)$, along with the friction coefficient $C_{f}$. In Figure 4, the RANS results are compared with the LES data for AOA $=5^{\circ}$, which is discussed in Ref. [30]. The LES were performed in a 3D domain based on a 2D extruded geometry. The agreement between the RANS and the reference well-resolved LES shows that the current CFD RANS can be used to truthfully represent free-flight conditions.
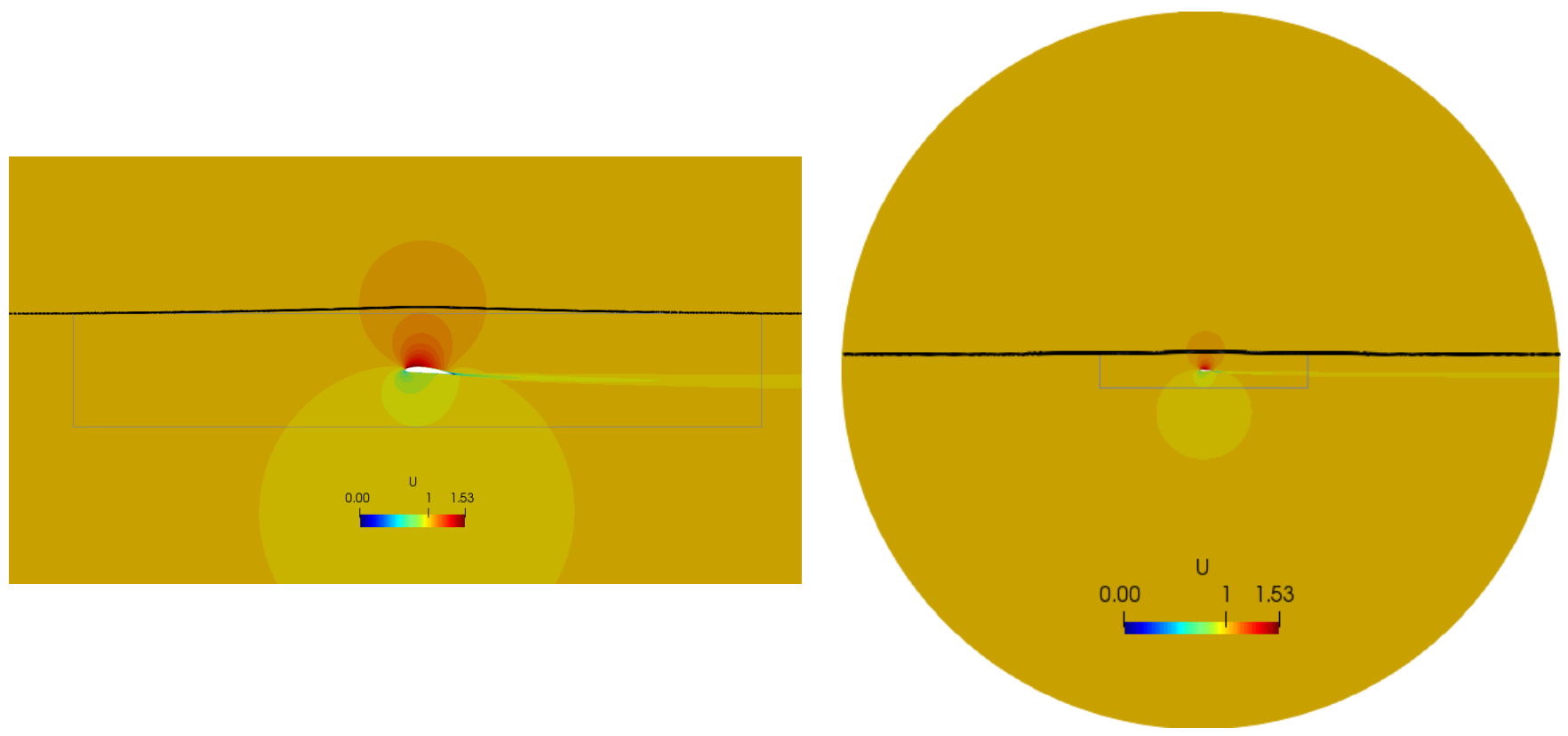

Figure 3. Left: Streamlines extracted from the unconfined simulation, at the location of the sidewalls in the wind tunnel test section. Right: Streamwise velocity distribution in the unconfined domain simulated via RANS. The box corresponds to the dimensions of the wind tunnel test section. 


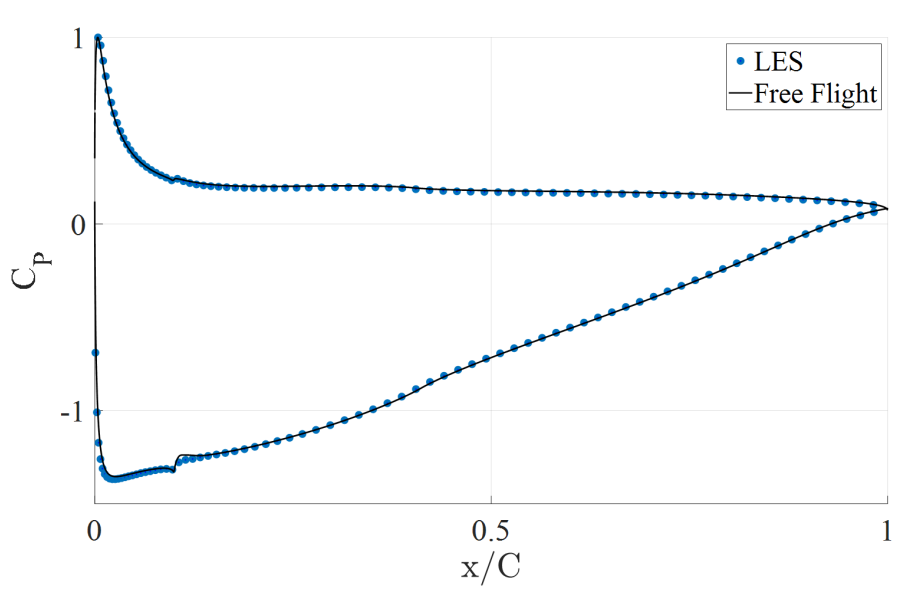

(a) $C_{p}$

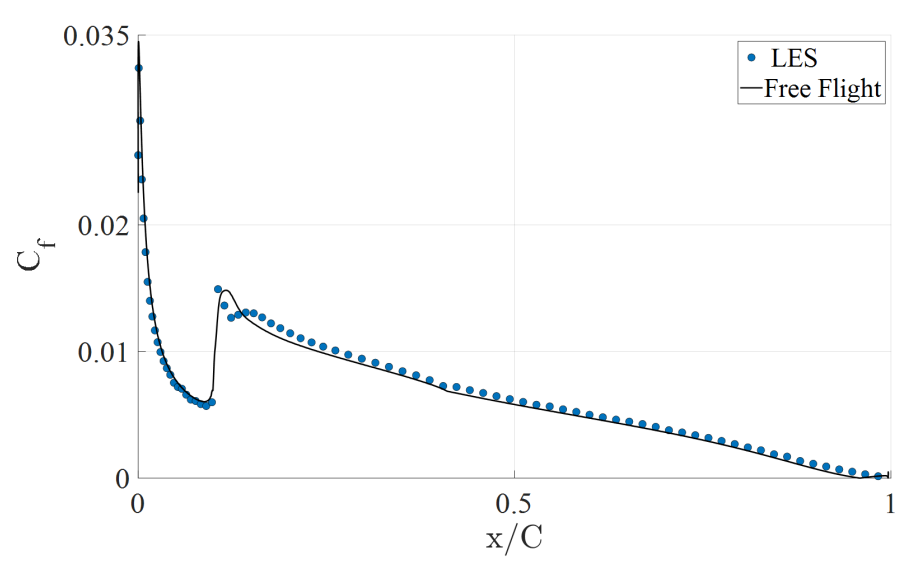

(b) $C_{f}$

Figure 4. LES vs. RANS: Chord-wise distribution comparisons at $\mathrm{AOA}=5^{\circ}$ in free-flight conditions. The well-resolved LES statistics were obtained from Ref. [30].

\subsection{Confined Wing in MTL Test Section}

With the simulation configuration mentioned in Section 2, simulations are implemented for various angles of attack for both $2 \mathrm{D}$ and 3D. In order to validate the setup with severe conditions, the selected angles of attack should be close to the stall angle. Two arbitrary AOAs are selected to present the results: $8^{\circ}$ and $11^{\circ}$. Since the stall angle for the NACA4412 wing section is around $14^{\circ}[20,31], \mathrm{AOA}=11^{\circ}$ represents near-stall conditions. The wing is mounted vertically in the tunnel and the static pressure is measured at the mid-height section. The same section is considered in the simulations, as in Figure 5. It also represents the section for 2D simulations with one layer of cells.

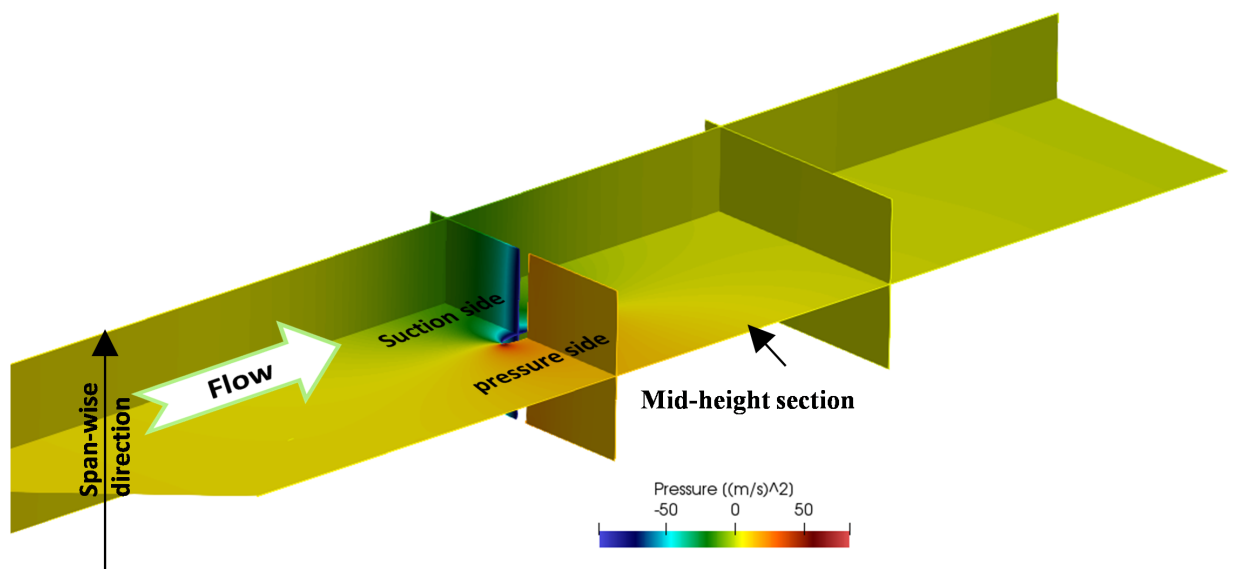

Figure 5. Pressure distribution in several cross-sections within the test section and around the airfoil.

The $C_{p}$ distribution is plotted over the suction and pressure sides at the aforementioned angle of attack in Figure 6. Although near-stall conditions are challenging for RANS turbulence models, the high suction peak is captured correctly for both angles of attack. The results show that the current CFD RANS setup can accurately reproduce the experimental pressure measurements. According to Section 2.1 and the current section, it can be concluded that our RANS setups, both for the wind-tunnel and free-flight configurations, are successfully validated and agree with independent reference data in both cases. 


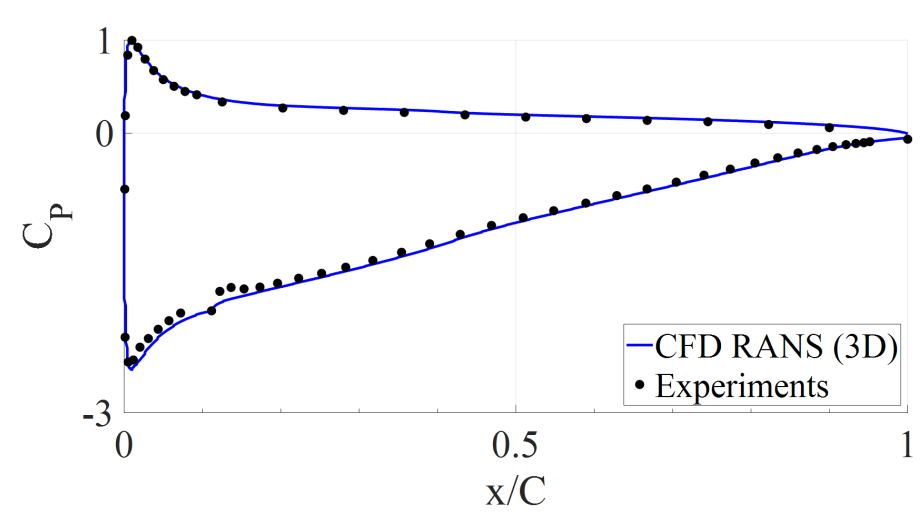

(a)

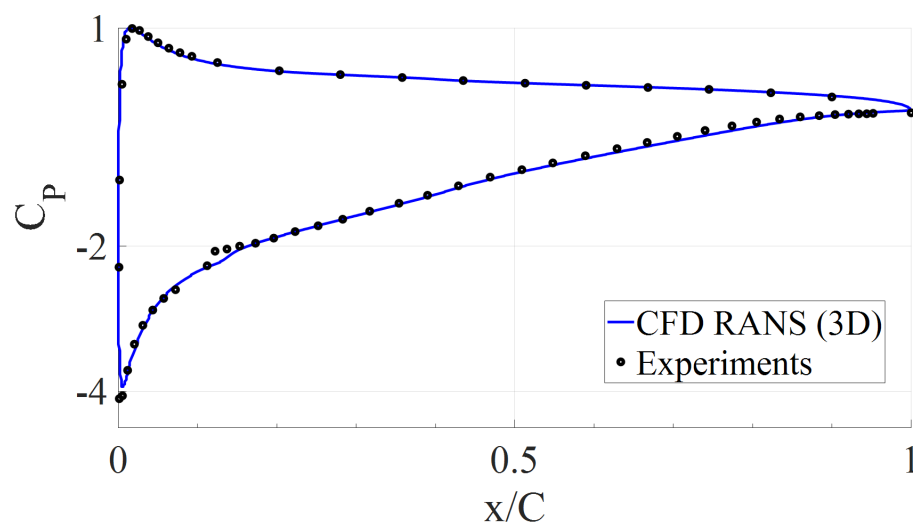

(b)

Figure 6. Chord-wise pressure coefficient distribution at mid-height wing section; (a) $\mathrm{AOA}=8^{\circ},(\mathbf{b}) \mathrm{AOA}=11^{\circ}$. The results are from the the simulation of the 3D domain.

\section{Methodology}

In this section, first we describe the justification for the proposed approach in this paper and then the method is described along with a few sample cases to show the efficiency of the method.

\subsection{Background of Linear Insert}

The objective in the well-known streamlining method is to convert the panel sidewalls into streamlined walls such that the wall inserts (WI) replicate the unconfined/free streamlines (FS) after subtracting the BL growth (BG), i.e., $W I-B G=F S$. There are two components in this method, which are the viscous and confinement effects, where the latter refers to the alteration of the free streamlines. These can be counted as the two main effects of wall interference in a subsonic wind tunnel, cf. Refs. [32-34]. It is known that both the free streamlines and the BL growth, have nonlinear trajectories, which imposes much complexity to the design of the ideal sidewalls. However, such an accuracy does not seem to be required in the case of moderate wall-interference as it is the case in subsonic wind tunnels and for low blockage ratios, as the streamlining method is not normally applied for these cases. Seeking for a reasonable solution for reducing wall interference in subsonic wind tunnels, this research studies the possibility of a reduced-order approximation of streamlined inserts.

Therefore, in the following two sections, we investigate the streamline confinement and viscous effect, respectively, in order to evaluate the deviation from a linear trajectory and whether they may be represented by a lower-order approximation. It should be noted that the presented data in the current section are shown to support the idea behind the proposed approach in Section 4.2 for reduced-order approximation.

\subsubsection{Streamline Confinement}

Confinement of the streamlines is the first direct effect of wind tunnel walls, as the sidewalls affect the streamlines' path. A 'solid blockage effect' is commonly observed in wind tunnel testing, and it causes an increase in the local velocity in the test Section [35]. In the case of a wake flow, 'wake blockage' is another effect caused by the streamline confinement inside the tunnel [36]. As discussed by El-Sherbiny [33], "The velocity outside the wake increases as a result of wake blockage so does the effective increase in velocity past the body location". Figure 3-right shows the streamwise velocity field in the unconfined domain obtained through RANS. Figure 3-left shows the streamlines from the unconfined simulation at the location of the sidewalls in the corresponding wind tunnel test section.

The extracted streamlines are analyzed in more detail in Figure 7. The maximum deflection is about $12 \%$ of the chord length, which equals $5 \%$ of the tunnel width. Although the streamlines are curved, we decide to approximate it with an equivalent piece-wise 
linear curve. In particular Figure 7 shows that the top streamline can be approximated with a straight line extending from the tunnel inlet to the trailing edge, and then another linear function can approximate the rest of the streamline up to the tunnel outlet.

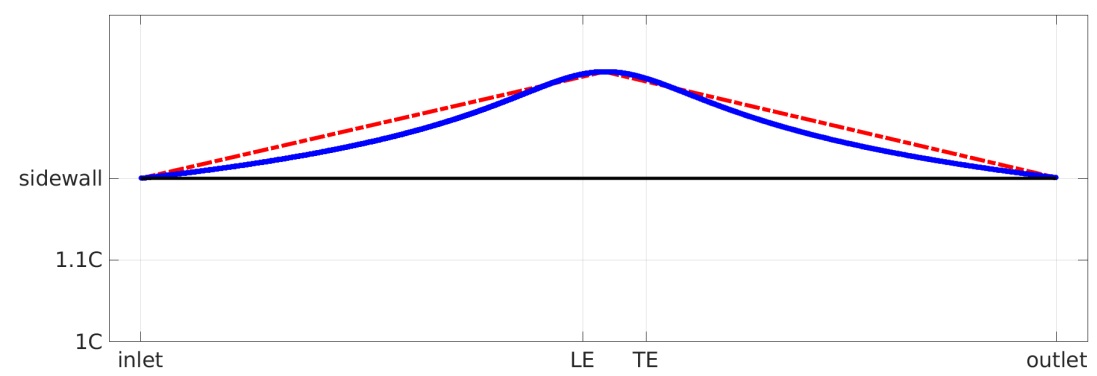

Figure 7. Flow streamlines in the unconfined domain at the equivalent location of wind tunnel top wall; blue: the original curves, red: fitted piece-wise linear curves. Root mean square error of each linear fitting $0.58 \% \mathrm{C}$. Physical aspect ratio of the plot is not preserved. LE and TE denote wing leading edge and trailing edge, respectively, which represent the position of the wing inside the tunnel.

\subsubsection{Viscous Effects}

In addition to the wall confinement, the BL growth on the sidewalls also affects the flow, through acceleration of the irrotational core. Although the BL growth on a flat plate is not linear, a linear function may be used to approximate the growth. Figure 8 shows the experimental results for the boundary-layer thickness, $\delta_{99}$, with respect to the streamwise direction over the wind-tunnel floor, where there is no model in the wind tunnel. The yellow line represents a linear fit to the data. As apparent, a linear approximation can well represent the BL growth, excluding the wake region (i.e., from the trailing edge (TE) to the tunnel outlet).

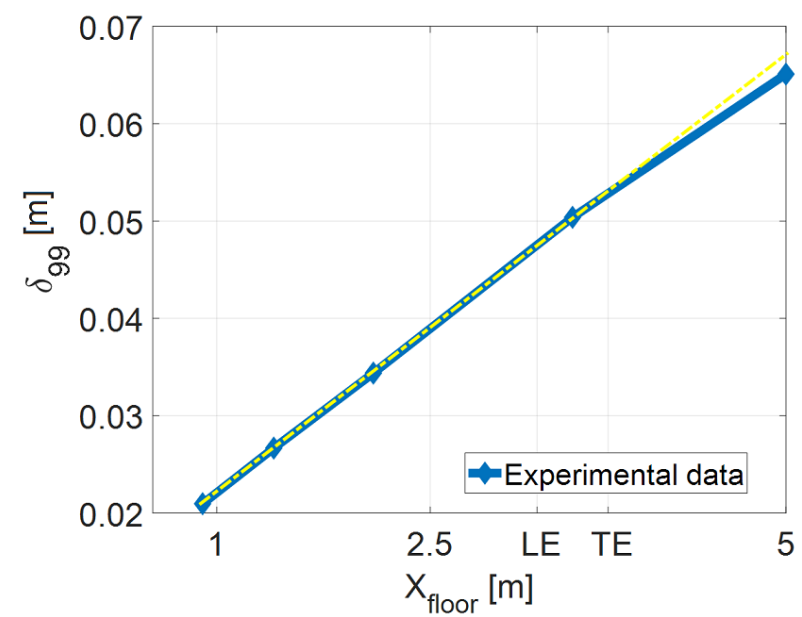

Figure 8. Boundary-layer growth with respect to the stream-wise direction over the wind tunnel floor; The yellow line is a linear function fitted to the boundary layer growth.

It is not clearly known how far the BL growth is blocking the flow path at a certain section in the presence of the airfoil. Different integral parameters are commonly used to approximate the narrowing extent due to the BL growth, however, neither of these truthfully resembles the blockage magnitude in the presence of a model in a wind tunnel. This explains why iterative procedures have been used in wall-correction methods. The effect of BL growth, i.e., wall-shear effects, will be individually assessed (via zero-shear wall) for different cases further in this paper.

Figure 9 illustrates a method to determine the boundary-layer edge. The considered measure to approximate the BL evolution is based on the turbulence content inside the BL 
when it reaches the outer flow. The separating edge is visible between the BL and the outer flow via the turbulence contour-plot in Figure 9-left. Regardless of the exact extent of the viscous-influence, the growth pattern can be observed, which is approximated with a slope line in Figure 9-right. More accurate approximation can even be obtained with a two-pieces linear function (on each side), the second one starting at the trailing edge of the airfoil.
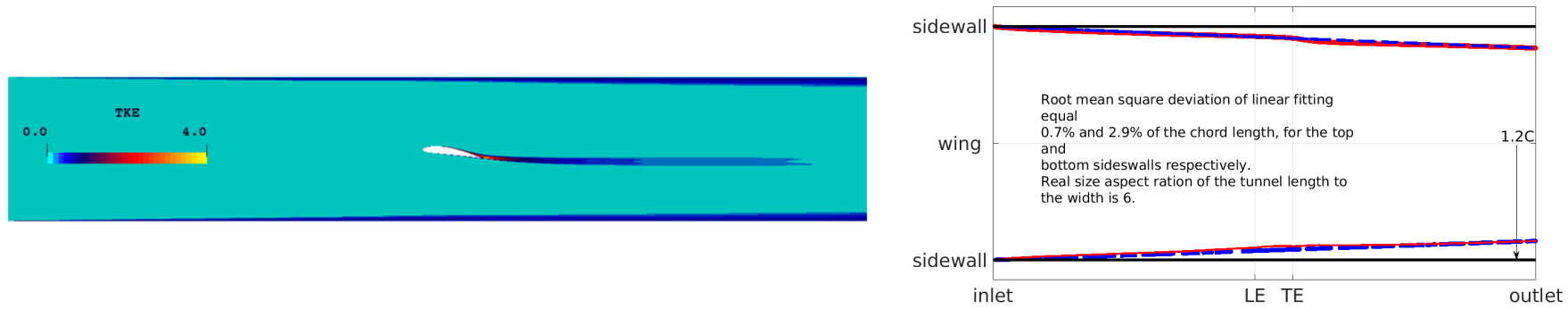

Figure 9. Boundary-layer edge with the presence of the airfoil at $\mathrm{AOA}=8^{\circ}$ according to turbulent kinetic energy; left: TKE contour within the specified range $[\mathrm{m} / \mathrm{s}]^{2}$, right: Contour line of TKE (red) and the linear fit to that (blue).

Analyzing the streamwise distributions and the BL growth reported in Figures 7 and 9, respectively, it can be seen that the inserts should be designed to produce a diverging test section.

\subsection{Numerical Approach}

According to the basics of insert-design methods, inserts should be defined such that the flow streamlines resemble those in the unconfined case after accounting for the viscous effects, i.e., the BL growth. The results above show that both the streamlines and the streamwise evolution of the BL thickness can be approximated by linear functions. Thus, here we propose to design the inserts based on linear profiles. Specifically, if the focus of the analysis is not the airfoil wake, then a single linear function can approximate both the BL growth and the streamlines. To investigate the feasibility of this idea, a numerical approach is considered, as discussed in the current section.

In order to design the inserts following a linear function, we consider a continuously diverging section, where the outlet width is unchanged and the inlet width is reduced by $8-24 \% \mathrm{C}$, where both top and bottom walls are inclined. The schematic of the suggested sidewalls is shown in Figure 10.

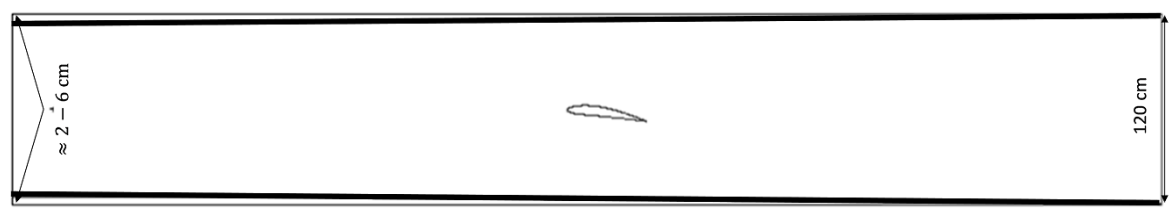

Figure 10. Schematic representation of the linear inserts.

In this section, we present our approach via the comparison between the free-flight condition and the wind-tunnel data with and without the inserts according to the chordwise pressure distribution. The pressure quantities are non-dimensionalized with respect to the dynamic pressure and the maximum for the resultant $C_{p}$ curve will be unity, see Equation (3). It is observed that the general effect of the test-section with parallel walls is a $C_{p}$ reduction; a larger peak at the suction side and a lower pressure at the pressure-side. This effect is shown in Figure 11a, which indicates that the effect in the 3D simulations (including the four wind tunnel walls) is more prominent than the 2D cases. In this figure, we show the simulation results with the original parallel walls, and with the modified diverging walls. In Figure 11b, the results are shown for the mid-height section of the 3D domain. The same inserts, with the same slope and configuration, are mounted as 3D sidewalls, extending along the wing span in a 3D domain, see Figure 1. The ceiling and 
floor of the wind tunnel are unchanged. The resulting pressure distribution is compared with that of the original test section. The wall-interference effect is substantially removed with the 3D linear inserts. The improvement is promising, and a complete removal of the wall-interference effect would require several additional iterations on the insert geometry.

The NACA4412 airfoil was analyzed at $\mathrm{AOA}=8^{\circ}$ in the simulated wind tunnel with the linear inserts. Figure $11 \mathrm{c}$ shows the $C_{p}$ distributions corresponding to the parallel-wall original wind tunnel in comparison with the free-flight condition, as well as the modified walls. General improvement with the inserts is observed, a fact that supports the feasibility of the proposed approach.

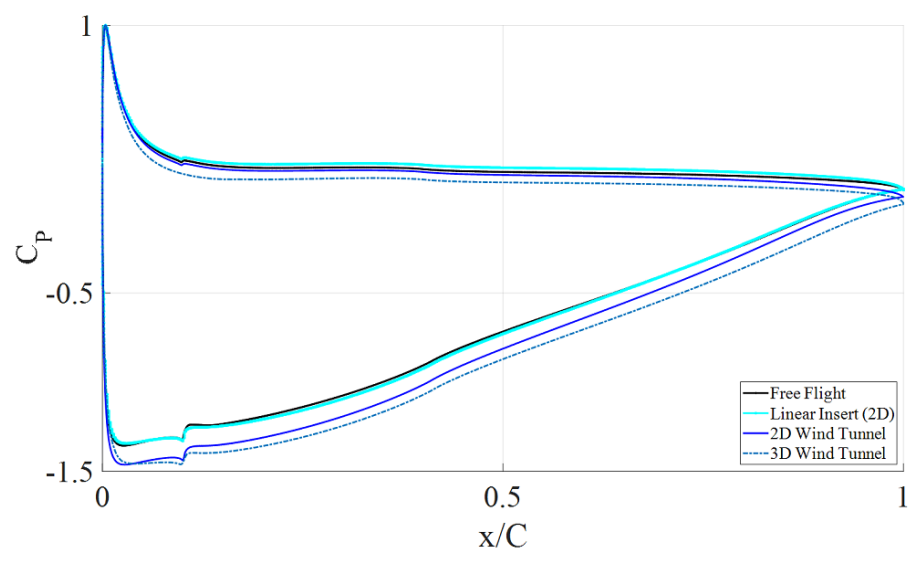

(a) 2D Linear; $\mathrm{AOA}=5^{\circ}$.

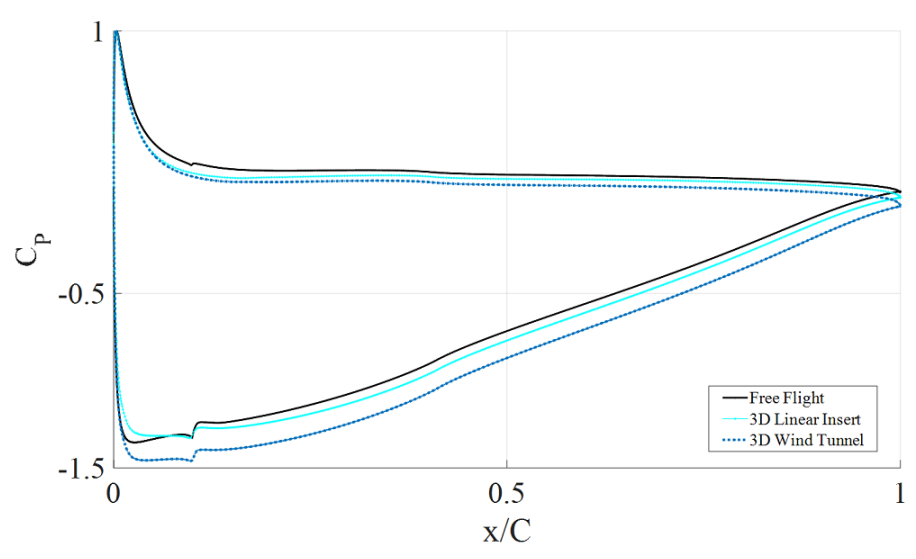

(b) $3 \mathrm{D}$ linear; $\mathrm{AOA}=5^{\circ}$.

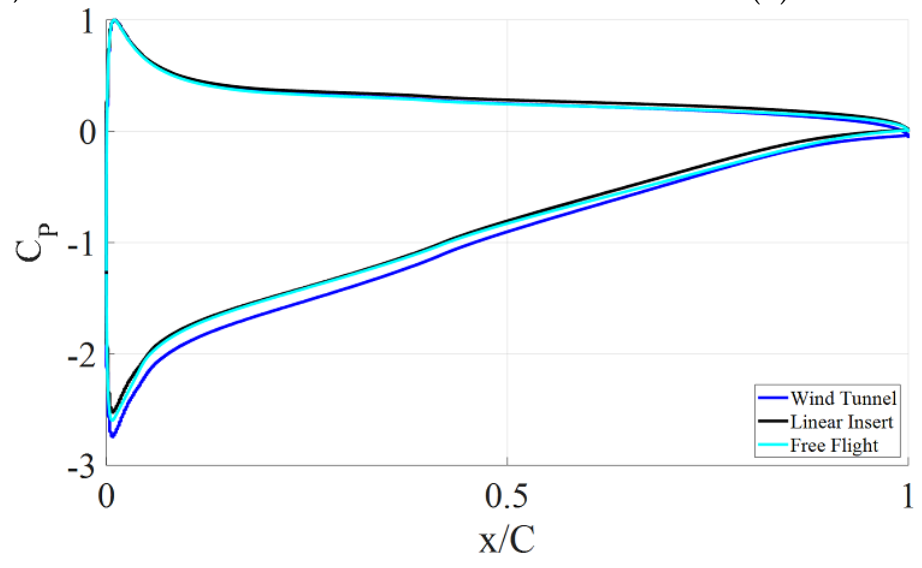

(c) $2 \mathrm{D}$ Linear; $\mathrm{AOA}=8^{\circ}$.

Figure 11. Effect of linear insert on $C_{p}$. Note that the parallel-wall tunnel is denoted by 'Wind Tunnel'.

A more detailed analysis of Figure $11 \mathrm{c}$ reveals that the $C_{p}$ compensation on the suction side is quite good over the first $65 \%$ of the $x / C$ range. However, at the last $30 \%$, i.e., $0.7<x / C<1$, the slope of $C_{p}$ is not well adapted. Note that the slope of the plotted curve shows the axial pressure gradient. Such observations reveal that a comprehensive aerodynamic evaluation is not possible solely based on $C_{p}$. In the next section, we will consider the comparison of the integrated aerodynamic forces, i.e., lift and drag.

\section{Results and Discussions}

With the method described in Section 4.2, few iterations were carried out for each case in order to find an appropriate configuration in which the deviation is decreased with respect to free-flight conditions. The goal is to find the initial thickness of the insert for the top and bottom walls, i.e., $\Delta_{\text {top }}$ and $\Delta_{\text {bot }}$, respectively, leading to a lower deviation between free-flight and wind tunnel model regarding the aerodynamic properties. Note that the presented results do not necessarily show the optimum configurations; trial and error can continue to improve the inserts especially at $\mathrm{AOA}=0^{\circ}$. The schematic 
representation of the $\Delta$ values shown in Figure 12 are non-dimensionalized with $w$ (which is the original tunnel width), and the resulting values are reported in Table 2. Two diverging states are assessed according to Figure 12-left and a half-diverging profile according to Figure 12-right. With using insert\#1, we tested the efficiency of the identical insert characteristics for all AOA.

Table 2. Insert configuration for each study case: three different cases are considered. Sketches are shown in Figure 12, where \#1 and \#2 are according to Figure 12-left and \#3 refers to Figure 12-right.

\begin{tabular}{ccccccc}
\hline & \multicolumn{6}{c}{$\Delta / \mathbf{w}$} \\
\cline { 2 - 6 } AOA [deg] & \multicolumn{2}{c}{ \#1 Diverging } & \multicolumn{2}{c}{ \#2 Diverging } & \multicolumn{2}{c}{ \#3 Half-Diverging } \\
\cline { 2 - 6 } & Top & Bot & Top & Bot & Top & Bot \\
\hline 0 & 4.8 & 3.5 & 3.8 & 2.6 & - & - \\
\hline 5 & 4.8 & 3.5 & - & - & - \\
\hline 8 & 4.8 & 3.5 & 4.8 & 1.7 & 2.7 \\
\hline 11 & 4.8 & 3.5 & 2.7 & 1.4 & 1.4 \\
\hline
\end{tabular}
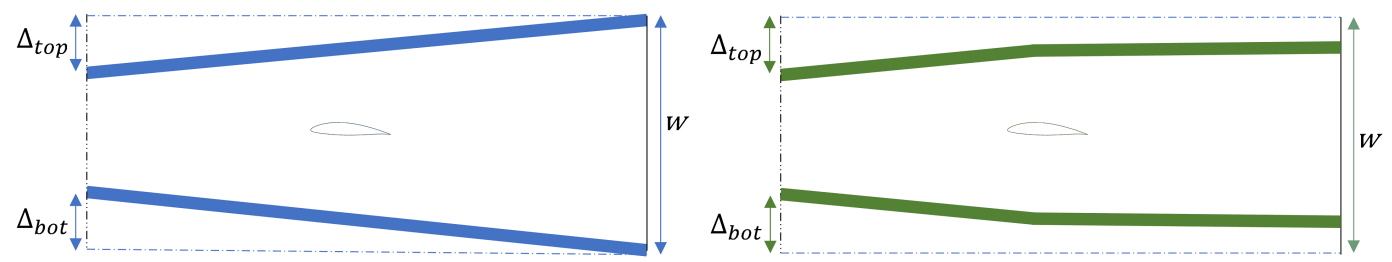

Figure 12. Schematic representation of two insert configurations: (left) completely diverging, and (right) half diverging with the breaking point at $x / C=0.5$.

In order to have a more complete comparison between free-flight conditions and the flow inside the wind tunnel, error parameters are defined based on the aerodynamic coefficients, i.e., lift coefficient $C_{L}$ and drag coefficient $C_{D}$. Although these two parameters represent some of the aerodynamic characteristics of the airfoil, their correctness does not necessarily imply a correct pressure distribution. As an example, equal deviations on the suction side and on the pressure side can result in a correct lift magnitude, while there would be a deviation in the pressure distribution. To address such deviations, additional indicators are considered: the value of $C_{p}$ at $x / C=0.5$ on both the suction and the pressure sides. These are selected as measures for a shift in the $C_{p}$ curve.

For each of the criteria, error values are calculated as the deviation with respect to the reference quantity, which is the value in free-flight conditions. Lift and drag deviations are scaled with the reference lift and drag values at the related free-flight condition, respectively. The $C_{p}$ deviations are as well scaled with the unit $C_{p}$ at the stagnation point. The resulting values can be interpreted as the deviation of static pressure, $\Delta p$, in terms of a percentage of $P_{d}$. Negative errors imply that the reference value (free-flight) is smaller than the wind tunnel value. The investigated cases are the airfoil at $\mathrm{AOA}=0^{\circ},+5^{\circ},+8^{\circ}$ and $+11^{\circ}$. Each case is assessed in the wind tunnel with parallel walls, and compared with the conditions of the unconfined flow. An additional virtual scenario is defined in which the flow is confined, but there is no shear on the wall and consequently no BL is formed. In this way the effect of confinement is investigated separately, see Section 4.1.1. This case is shown as "zero-shear wall" in the plots. As already discussed, inserts are considered to modify two sidewalls in the wind tunnel. The main configuration is the one-piece linear, while the two-pieces linear configuration is also tested, as shown in Figure 12. 


\subsection{Lift}

To initiate the aerodynamic analysis, we compare the lift coefficients from freeflight conditions with that obtained in the wind tunnel, with the various insert designs, in Figure 13. The $C_{L}$ deviation due to wall interference is stated as the percentage of the reference lift coefficient, $\Delta C_{L} / C_{L}$ (denoted as normalized deviation) in subplot a. Since the lift value has a lower magnitude at the lower AOA, the non-scaled $C_{L}$ deviation is also shown in Figure $13 \mathrm{~b}$ as $\Delta_{C_{L}}$.

The wind tunnel walls lead to an over-prediction of the lift, particularly at lower AOA. Looking at Figure 13a, at higher angle of attack, the normalized deviation decreases so that the lift error due to wall-interference is only $3.5 \%$ near-stall $\left(\mathrm{AOA}=11^{\circ}\right)$. However, $\Delta_{C_{L}}$ has a minimum magnitude at zero AOA; the deviation of the lift force is $\approx 3.5 \%$ of $P_{d}$ over unit of the airfoil surface. At AOA $=8^{\circ}$ and with the panel sidewalls $\approx 7.5 \%$ of $P_{d}$ is added to the lift value due to the wall interference. Note that the mentioned value is for each $\mathrm{m}^{2}$ of the airfoil surface.

We also consider zero-shear walls to assess the effect of the BL growth, i.e., the observed error in the zero-shear cases is solely due to the confinement effect. At lower angles of attack, where the blockage ratio is even smaller, the confinement effect is bigger compared to the near-stall cases. It may be due to the fact that the streamlines are inclined downward at higher AOA, which is closer to the downward path of the flow passing behind the top sidewall (due to the BL growth). This is even true if one compares the non-normalized $C_{L}$ deviations at 0 and $11^{\circ} \mathrm{AOA}$. At $\mathrm{AOA}=0^{\circ}$, viscosity is responsible for only $\approx 25 \%$ of wall-interference, while it is $\approx 80 \%$ at $\mathrm{AOA}=11^{\circ}$. This is visible when comparing the red and blue bars, where for, e.g., at $\mathrm{AOA}=0^{\circ}$ the error of zero-shear wall is $75 \%$ of the original wall, indicating that removing the shear from the wall only eliminates $25 \%$ of the deviation.

With a simple linear insert in a wind tunnel, the lift deviation from the free-flight condition is almost removed so that the accuracy of the wind tunnel modeling is $97 \%$ in the whole AOA range under study. At near-stall AOA, using the half-diverging insert in a wind tunnel can result in almost the same lift value as in free-flight conditions.

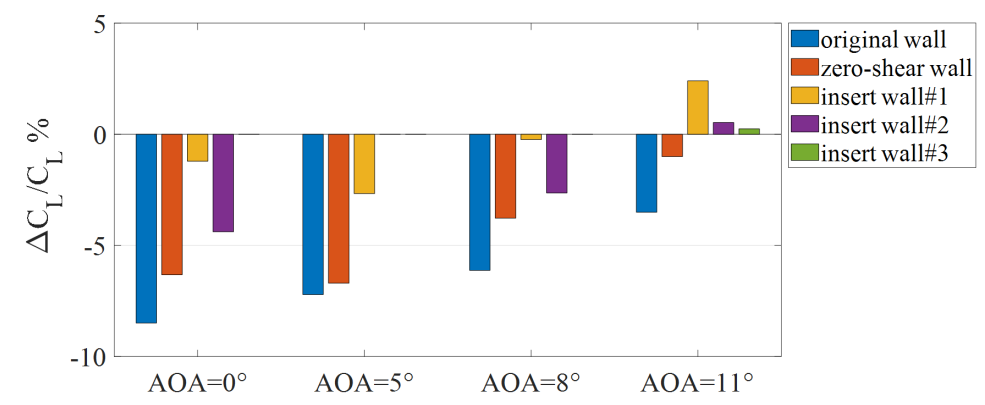

(a) Scaled $C_{L}$ deviation with respect to the local $C_{L}$.

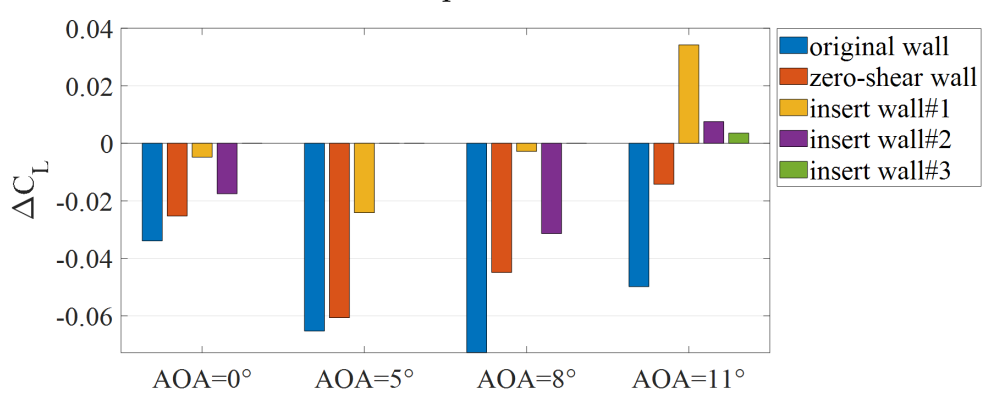

(b) Deviation of $C_{L}$ ( Lift deviation as a fraction of $P_{d}$ over a unit exposed-surface).

Figure 13. Relative deviation between the lift in free-flight conditions and that in the wind tunnel, using various insert designs. The insert wall numbers are according to Table 2 and the number of bars may differ for different AOA. 


\subsection{Drag}

Increasing the $\mathrm{AOA}$ in a wind tunnel leads to increasing deviation in drag with respect to the free-flight conditions. Figure 14 shows the $\Delta C_{D}$ deviation, as well as $\Delta C_{D} / C_{D}$. Far from stall $\left(\mathrm{AOA}=0-8^{\circ}\right)$, the wind tunnel drag values can represent the free-flight drag with a deviation of less than $4 \%$, while the deviation from free-flight conditions increases to $12 \%$ near-stall. With the linear inserts, the drag deviation is still relatively small for most cases. A half-diverging insert will reduce the disagreement of drag down to $4 \%$ even in near-stall conditions $\left(\mathrm{AOA}=11^{\circ}\right)$. At $\mathrm{AOA}=8^{\circ}$ a simple linear insert makes the drag equal to that of the free-flight conditions. Additional iterations are required to reduce the deviation with respect to free-flight conditions at zero incidence angle.

Figure $14 \mathrm{~b}$ shows the level of deviation due to the wall-interference as a factor of $P_{d}$ for a unit exposed-surface. With the panel sidewalls and at near stall, the wall interference in drag is almost 0.0042 of $P_{d}$, which can decrease to 0.0015 of $P_{d}$.

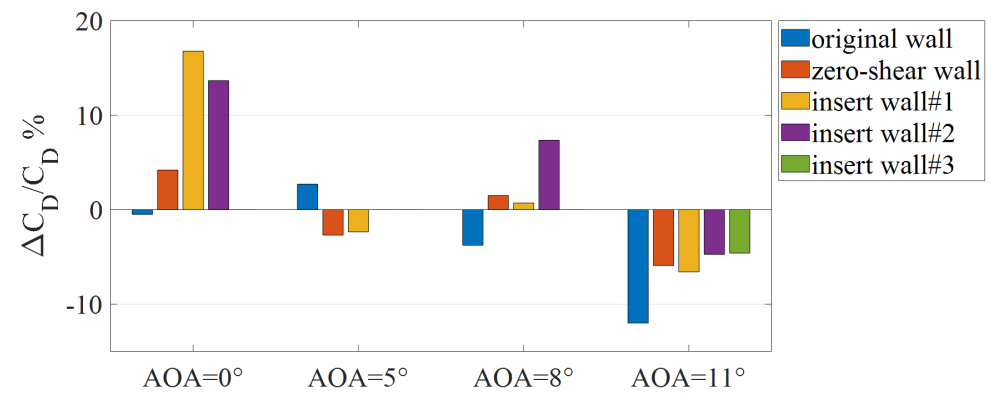

(a) Scaled $C_{D}$ deviation with respect to the local $C_{D}$.

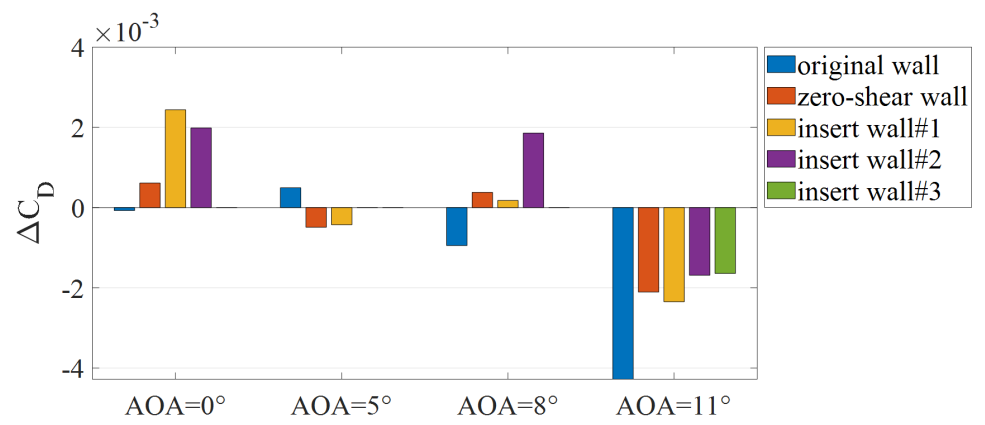

(b) Deviation of $C_{D}$ (Drag deviation as a factor of $P_{d}$ over a unit exposed-surface).

Figure 14. Relative deviation between the drag in free-flight conditions and that in the wind tunnel, using various insert designs.

\subsection{Local $C_{p}$}

The value of $C_{p}$ at $x / C=0.5$ on the suction side is investigated in Figure 15a. The deviation due to parallel walls is about $7 \%$, an error which decreases to $4 \%$ near-stall. The percentages are in terms of unit $C_{p}$ at the stagnation point. With the application of diverging inserts, this deviation is reduced to $2 \%$ far from stall. Half-diverging inserts can reduce the deviation with respect to free-flight conditions down to $2.5 \%$ near the stall angle.

As shown in Figure 15b, the wall-interference on the pressure side is almost negligible at all AOA. Regardless of the use of inserts, the deviation is less than 3\%. With the application of inserts, the deviation remains below $\approx 3 \%$. Hence the pressure-side $C_{p}$ data in a wind tunnel is in a fair agreement with the free-flight conditions. 


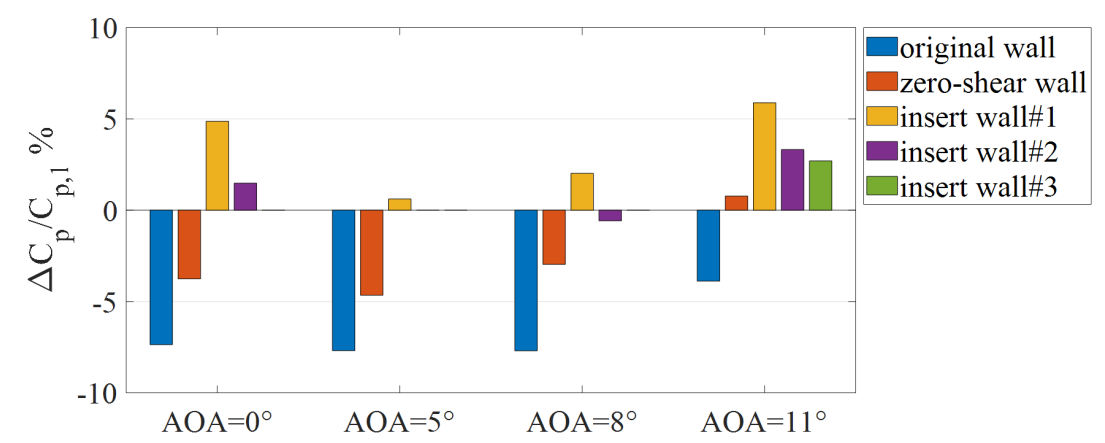

(a) Suction side

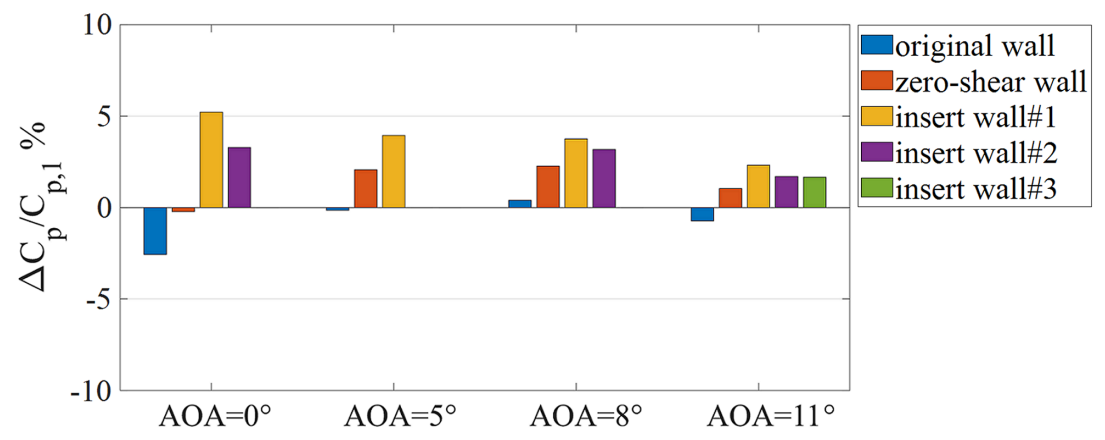

(b) Pressure side

Figure 15. Deviation of $C_{p}$ at $x / C=0.5$ between free-flight and experimental conditions, for (a) suction and (b) pressure sides, using various inserts.

\section{Conclusions and Outlook}

This work aims at devising a method to allow for wind-tunnel experiments replicating free-flight conditions, based on CFD for both situations. This may be relevant when one is interested in having the same pressure distribution over a wing in a wind tunnel, e.g., for control, noise, icing, etc. In order to improve the accuracy of wind tunnel modeling, this study investigates the effect of inclined sidewalls via a numerical sensitivity analysis. The side wall alignment is hence used as a calibration parameter to reduce wallinterference effects. With the goal of achieving a better realization of real, i.e., unconfined, free-flight conditions, wall-interference effects can be reduced even for sub-sonic flows under low / moderate blockage ratios.

A numerical study is implemented based on CFD RANS to model the MTL wind tunnel at KTH as a test case. Over a wide range of AOAs including near-stall conditions, the CFD model reproduced the same chordwise pressure distribution as measured in the wind tunnel. The confinement effect is investigated, as well as the shear effects of the sidewalls. Their impact on aerodynamic quantities is assessed both in terms of the lift and drag coefficients, as well as the local pressure coefficient.

The influence of sidewall BLs is found to vary with AOA, while neither the confinement, nor the viscous effect correlate directly with the AOA. Furthermore, the viscous effect is not the dominant interference factor for lift calculations at lower AOAs, instead confinement plays the main role. Drag evaluation is hardly affected by wall-interference for the conditions far from stall, while lift is overestimated by $\approx 3.5-8.5 \%$ depending on the AOA. In the wind tunnel model the $C_{p}$ values on the pressure side of the wing corresponds accurately to free-flight conditions, while the suction side deviates approximately $7 \%$ at the mid-chord.

A simple method with linear sidewalls is outlined to reduce wall-interference. Our results indicate that a linear estimation of the surrounding streamlines and the BL growth can improve the agreement between wind tunnel data and free-flight conditions. Accordingly, two linear configurations are proposed and tested over the range of AOAs. The functionality of the methodology is encouraging since only few iterations are required; the 
deviation between wind tunnel results and free-flight conditions can be reduced to below $1 \%$ for aerodynamic parameters. Consequently the wind tunnel data can fairly represent free-flight conditions through the use of linear inserts. The main advantage of such a method is hence its simplicity in the implementation, compared to the more cumbersome method of streamlining. Although the method is applied and tuned for each AOA individually, the wall-effect is decreased with a same linear insert even without specific tuning for the AOA.

According to the presented sensitivity analysis, the design process is planned to include all the confining walls in the wind tunnel, including the floor and ceiling, in order to realize free-flight conditions in a wind tunnel. An optimization can complement the design process for the construction and test of the inserts. The gap between the free-flight model and the corresponding wind tunnel model can then, e.g., be minimized by changing the configuration of the inserts.

Author Contributions: Conceptualization, N.T. and P.S.; methodology, N.T., P.S. and R.V.; software, N.T.; validation, N.T., R.Ö. and R.V.; formal analysis, R.Ö.; investigation, N.T.; resources, P.S. and R.Ö.; data curation, N.T.; writing—original draft preparation, N.T.; writing—review and editing, N.T., R.Ö. and R.V.; visualization, N.T.; supervision, P.S.; project administration, N.T.; funding acquisition, P.S. All authors have read and agreed to the published version of the manuscript.

Funding: Financial support provided by the Knut and Alice Wallenberg Foundation is gratefully acknowledged. The computations/data handling/[SIMILAR] were/was enabled by resources provided by the Swedish National Infrastructure for Computing (SNIC), partially funded by the Swedish Research Council through grant agreement no. 2018-05973.

Acknowledgments: The authors are grateful for all the data made available by the other researchers referenced in the text.

Conflicts of Interest: The authors declare no conflict of interest. The funders had no role in the design of the study; in the collection, analyses, or interpretation of data; in the writing of the manuscript, or in the decision to publish the results.

\section{References}

1. Vinuesa, R.; Rozier, P.H.; Schlatter, P.; Nagib, H.M. Experiments and Computations of Localized Pressure Gradients with Different History Effects. AIAA J. 2014, 52, 368-384. [CrossRef]

2. Sanmiguel Vila, C.; Vinuesa, R.; Discetti, S.; Ianiro, A.; Schlatter, P.; Örlü, R. Experimental realisation of near-equilibrium adverse-pressure-gradient turbulent boundary layers. Exp. Therm. Fluid Sci. 2020, 112, 109975. [CrossRef]

3. Sanmiguel Vila, C.; Vinuesa, R.; Discetti, S.; Ianiro, A.; Schlatter, P.; Örlü, R. Separating adverse-pressure-gradient and Reynoldsnumber effects in turbulent boundary layers. Phys. Rev. Fluids 2020, 5, 064609. [CrossRef]

4. Romano, D.; Alfredsson, P.; Hanifi, A.; Örlü, R.; Tillmark, N.; Borodulin, V.; Ivanov, A.; Kachanov, Y.; Minervino, M. Design and Tests of Wind-Tunnel Sidewalls for Receptivity Experiments on a Swept Wing. In Applied Mechanics and Materials; Trans Tech Publications Ltd: Tokyo, Janpan, 2013; Volume 390, pp. 96-102.

5. Rangarajan, D.; Curtis, J.S. The Effect of Spanwise Width on Rectangular Jets With Sidewalls. J. Fluids Eng. 2012, 134, 031202. [CrossRef]

6. Ljungskog, E.; Sebben, S.; Broniewicz, A. Flow Angularity Investigations in an Automotive Slotted Wall Wind Tunnel. Energies 2019, 12, 4575. [CrossRef]

7. Korvin-Kroukovsky, B. On high speed wind tunnels. J. Frankl. Inst. 1939, 227, 461-471. [CrossRef]

8. Wolf, S.W. Adaptive wall technology for improved wind tunnel testing Techniques-A review. Prog. Aerosp. Sci. 1995, 31, 85-136. [CrossRef]

9. Dobriyal, R.; Mishra, M.; Bölander, M.; Skote, M. Effects of Streamlining a Bluff Body in the Laminar Vortex Shedding Regime. J. Fluids Eng. 2019, 142, 024501. [CrossRef]

10. Stafford, L.G. A streamline wind-tunnel working section for testing at high blockage ratios. J. Wind Eng. Ind. Aerodyn. 1981, 9, 23-31. [CrossRef]

11. Wolf, S. Self Streamlining Wind Tunnel: Low Speed Testing and Transonic Test Section Design; NASA Contractor Report; Langley Research Center: Hampton, VA, USA, 1977.

12. Acum, W.E.A.; Garner, H.C.; Maskell, E.C.; Rogers, E.W.E. Subsonic Wind Tunnel Wall Corrections; AGARD AG-109; AGARD: Paris, France, 1966. 
13. Newman, P.; Anderson, E.; Peterson, J.; Aeronautics, U.S.N.; Scientific, S.A.; Branch, T.I. Aerodynamic Design of the Contoured Wind-Tunnel Liner for the NASA Supercritical, Laminar-Flow-Control, Swept-Wing Experiment; NASA TP-2335; National Information Branch: Tokyo, Japan, 1984.

14. Nagib, H.; Hites, M. High Reynolds number boundary-layer measurements in the NDF. In Proceedings of the 33rd Aerospace Sciences Meeting and Exhibit, Reno, NV, USA, 9-12 January 1995

15. Kao, Y.H.; Jiang, Z.W.; Fang, S.C. A Computational Simulation Study of Fluid Mechanics of Low-Speed Wind Tunnel Contractions. Fluids 2017, 2, 23. [CrossRef]

16. Meyer, O.; Nitsche, W. Update on progress in adaptive wind tunnel wall technology. Prog. Aerosp. Sci. 2004, 40, 119-141. [CrossRef]

17. Hyvärinen, A. Investigation of Blockage Correction Methods for Full-Scale Wind Tunnel Testing of Trucks. Master's Thesis, KTH, Stockholm, Sweden, 2015.

18. Haque, A.U.; Asrar, W.; Omar, A.A.; Sulaeman, E.; Ali, M.J. Comparison of data correction methods for blockage effects in semispan wing model testing. In European Physical Journal Web of Conferences; EDP Sciences: Ulis, France, 2016; Volume 114, p. 02129.

19. Hosseini, S.; Vinuesa, R.; Schlatter, P.; Hanifi, A.; Henningson, D. Direct numerical simulation of the flow around a wing section at moderate Reynolds number. Int. J. Heat Fluid Flow 2016, 61, 117-128. [CrossRef]

20. Coles, D.; Wadcock, A.J. Flying-Hot-wire Study of Flow Past an NACA 4412 Airfoil at Maximum Lift. AIAA J. 1979, 17, 321-329. [CrossRef]

21. Schlatter, P.; Örlü, R. Turbulent boundary layers at moderate Reynolds numbers: Inflow length and tripping effects. J. Fluid Mech. 2012, 710, 5-34. [CrossRef]

22. Lindgren, B.; Johansson, A.V. Evaluation of the Flow Quality in the MTL Wind-Tunnel; Technical Report TRITA-MEK 2002:13; Dept. of Mechanics, KTH: Stockholm, Sweden, 2002.

23. The OpenFOAM Foundation. OpenFOAM User Guide, Version 7. 2019. Available online: https://cfd.direct/openfoam/userguide-v7 (accessed on 27 May 2021)

24. Menter, F.R. Two-equation eddy-viscosity turbulence models for engineering applications. AIAA J. 1994, 32, 1598-1605. [CrossRef]

25. ANSYS, Inc. ICEM CFD User Manual, Release 14.0; ANSYS, Inc.: Canonsburg, PA, USA, 2011.

26. ANSYS, Inc. ANSYS ICEM CFD Help Manual, Release 18.2; ANSYS, Inc.: Canonsburg, PA, USA, 2017.

27. Geuzaine, C.; Remacle, J.F. Gmsh: A 3-D finite element mesh generator with built-in pre- and post-processing facilities. Int. J. Numer. Methods Eng. 2009, 79, 1309-1331. [CrossRef]

28. Vinuesa, R.; Hosseini, S.; Hanifi, A.; Henningson, D.; Schlatter, P. Pressure-Gradient Turbulent Boundary Layers Developing Around a Wing Section. Flow Turbul. Combust. 2017, 99, 613-641. [CrossRef] [PubMed]

29. Tabatabaei, N.; Vinuesa, R.; Schlatter, P. Techniques for turbulence tripping of boundary layers in RANS simulations. Flow Turbul. Combust. 2021, under review

30. Vinuesa, R.; Negi, P.; Atzori, M.; Hanifi, A.; Henningson, D.; Schlatter, P. Turbulent boundary layers around wing sections up to $R e_{c}=1,000,000$. Int. J. Heat Fluid Flow 2018, 72, 86-99. [CrossRef]

31. Moses, O.P.; Vincent, A.O. Computational Study of Aerodynamic Flow over NACA 4412 Airfoil. Br. J. Appl. Sci. Technol. 2017, $21,1-11$.

32. Mokry, M.; Chan, Y.; Jones, D.J. Two-Dimensional Wind Tunnel Wall Interference; Technical Report AGARD-AG-281; Advisory Group for Aerospace Research and Development, National Aeronautical Establishment, National Research Council: Ottawa, ON, Canada, 1983.

33. El-Sherbiny, S. Effect of Wall Confinement on the Aerodynamics of Bluff Bodies. Ph.D. Thesis, University of British Columbia, Vancouver, BC, Canada, 1972.

34. Modi, V.J.; Akutsu, T. Wall Confinement Effects for Spheres in the Reynolds Number Range of 30-2000. J. Fluids Eng. 1984, 106, 66-73. [CrossRef]

35. Ross, I. Wind Tunnel Blockage Corrections: An Application to Vertical-Axis Wind Turbines; University of Dayton: Dayton, OH, USA, 2010.

36. Barlow, J.; Rae, W.; Pope, A. Low-Speed Wind Tunnel Testing; Wiley: Hoboken, NJ, USA, 1999. 